\title{
Indices
}

Der Index verborum ist ein gemeinsamer Index für beide Inschriften (Act. Aug. und Act. Sev.); dabei wurden auch unvollständig überlieferte, in der Edition ergänzte Wörter berücksichtigt. Bei den Indices der Namen und Orte dagegen werden die Einträge nach Inschriften getrennt aufgeführt. Die beiden Indices nominum sind aufgrund der lückenhaften Überlieferung nicht alphabetisch sortiert, sondern werden nach Personengruppen und innerhalb dieser nach ihrem ersten Erscheinen in der Inschrift aufgelistet.

\section{Index verborum}

$\infty$ Act. Sev. 2 I 6

XXV Act. Aug. I7

XXVII Act. Aug. I47

CVIIII Act. Sev. I78, I84, I85(2), I87, 228

CX Act. Aug. I23, I25, I30

abesse Act. Aug. 27

abnepos Act. Sev. 55, 57, 72, 74, II2, II4, 2 IO, 21 $2,275,278$

abolere Act. Sev. 30, 77

acceptor Act. Sev. I3 I, I60

acceptrix Act. Aug. 97; Act. Sev. I3 If., I 34 f., I 4 I

accipere Act. Aug. 30, 35, 89; Act. Sev. 214, 2 I $5,216,252$

accitus Act. Sev. 165, I79

accusator Act. Sev. 45f., 77

Achivus Act. Aug. 91, I 19, I34; Act. Sev. I 8 I, 225

actus Act. Sev. 258, 259

addere Act. Sev. I9, I 40

adesse Act. Aug. AB 8, I9, 50, 58, I 50; Act.

Sev. 3I, 65, 238

adicere Act. Aug. 100, I 56, I60; Act. Sev.

I 5, I 45, I 78,2 I $2,216,233$

adimplere Act. Sev. 8

administrare Act. Sev. 79

administratio Act. Sev. 52

admonere Act. Sev. 7, 75, 78.

adnepos Act. Sev. 55, 57, 72, 74, II 2, II4, 2 I0, 2 I $2,275,278$

adolescentia Act. Sev. I 8

adpetere Act. Sev. I3 adscendere Act. Sev. 82, 97f., I00, I78, 234

adsistere Act. Sev. I 80, 224, 230, 283

adspergere Act. Sev. 167

adstare Act. Sev. I 2 I, I 84

adtribuere Act. Aug. AB 5, C 4,63

advocare Act. Sev. I 8

$\{$ a eborneus siebe eborneus

aedis Act. Aug. I0, 30, $3 \mathrm{I}(2), 32,37,70,74$;

Act. Sev. 60, 64, 68, 70(2), 80, 229, 234,

$25 \mathrm{I}$

aenator ( $\rightarrow$ aeneator) Act. Aug. 88

aeneator Act. Sev. 247

aerarium Act. Aug. $\mathrm{AB}_{4}, \mathrm{C}_{3}, 63$; Act. Sev.

29

aerius Act. Sev. 246

aeternitas Act. Sev. 23f.

agere Act. Aug. C I2(2); Act. Sev. 21, 24,

59

agna femina Act. Aug. 90, 93, 97f., 98; Act.

Sev. I 38 , I 4 I (2)

aheneus Act. Aug. 60, 62

alacer Act. Sev. 2 I 8

albus Act. Sev. I 8 I

aliter Act. Sev. I4

alius Act. Sev. 9, 234

alter Act. Aug. 6I, 62, I04

alveus Act. Sev. 67, 208, 269

ambo Act. Aug. 6I

amplius Act. Aug. 66

amplus Act. Sev. 5

annalis Act. Sev. I 2

annus Act. Aug. $\mathrm{AB}$ 2, $\mathrm{AB} 6, \mathrm{C}_{16}$, 17, 25 ;

Act. Sev. 7, I 3, I4, I 5, 20, 23, 28(2), 43 
antiquus Act. Aug. AB 2; Act. Sev. 256 antruare Act. Sev. 260

apparitio Act. Sev. 259

applicare Act. Sev. 69, 92

Aprilis Act. Aug. 37

aqua Act. Sev. I68, I78, 234

ara Act. Sev. I 55, I67, I77, 223, 229

aratrum Act. Sev. 244

area Act. Sev. 68, 80, 230

argenteus Act. Sev. 252

armiger Act. Sev. 246

arvicola Act. Sev. 239

asinarius Act. Sev. 248

ast Act. Aug. I25; Act. Sev. I85

atalla Act. Aug. I07, I 32

attentus Act. Sev. 2r 8f.

audientia Act. Aug. C I 5, I4

augere Act. Aug. 94, I27; Act. Sev. 20, I29,

I39, I 58, I73, I 82, I 86, 227, 232

auratus Act. Sev. 243

Ausonius Act. Sev. 238

auspicium Act. Sev. I2, I6

bene Act. Aug. $\mathrm{C}_{14}, 7$, IO

biduum Act. Aug. 89; Act. Sev. 260

biga Act. Sev. 21 5, 216, 248, 255, 257, 258

bini Act. Aug. 3, 35

bison Act. Sev. 219

bonum factum Act. Aug. 80

bonus Act. Aug. C I4, I I I, I43; Act. Sev. 9?, 22, 94

bos Act. Sev. I60, 164, I69

bos femina Act. Aug. I 19, I 22; Act. Sev. I 82

bos mas Act. Aug. 103, I06; Act. Sev. I57, I 63

caelum Act. Sev. 239

caerimonia Act. Aug. 86

campus Act. Aug. 90, 108; Act. Sev. 243

canere Act. Aug. 21, 88, I48; Act. Sev. 20, $235,237,260$

cantus Act. Sev. 237

capra femina Act. Aug. 91, 93, 98; Act. Sev. I38, I 4 I

carcer Act. Sev. 254, 257

carmen Act. Aug. 21, I48, I49; Act. Sev. 235, $25 \mathrm{I}, 260$

carus Act. Sev. 6I, 63

castra Act. Sev. 245

causa Act. Aug. 56, I I I; Act. Sev. 92, I 83 cavus Act. Sev. 244

celebrare Act. Aug. I I ; Act. Sev. 17, 29, 75 , I I I

celebritas Act. Sev. 7, 8

cella Act. Sev. I 84

cena Act. Sev. I 83

cenare Act. Sev. 260

censere Act. Aug. 34; Act. Sev. 47

centeni Act. Sev. 2 I 9

centensimus/centesimus Act. Aug. Ci6, 25 ; Act. Sev. 20

centum Act. Sev. 28, 43, 259

centum et $\mathrm{X}$ Act. Aug. IoI

certior Act. Aug. 87

cetera Act. Aug. 74, I06, I I 8, I 22, I37, I42; Act. Sev. I39, I58, I64, I73, I82, 227,232

ceteri Act. Aug. C I I; Act. Sev. 74, 82, I I4, I79, I80, I 83, 2I 2, 223, 228, 229, 230, $253,266,279,282$

chorus Act. Aug. 3, 21; Act. Sev. 25 I

circensis Act. Sev. 214, 217

circumducere Act. Sev. 253

circumire Act. Sev. 79

circus Act. Aug. 158, I6I; Act. Sev. 253, $254,267,303$

civis Act. Sev. 76

clarissimus Act. Sev. 26, 29

clarus Act. Sev. 29

cognoscere Act. Aug. 28

coire Act. Sev. 256

collega ( $\rightarrow$ conlega) Act. Aug. C s

collegium Act. Aug. C I 2, 29, 30, 40, 97, 99; Act. Sev. 6, I4, 43, 64, 67, 88, I 3 I, I 36, I39, I42, I63, I66, I67, 207

columna Act. Aug. 60, 6I

comitia Act. Sev. 6

commentarium Act. Aug. 59, 62; Act. Sev. I, 47

commissio Act. Sev. 220(2)

committere Act. Aug. 83, 85, 100, 108, I 56, I60; Act. Sev. 206, 267(3)

commone facere Act. Aug. 65

communis Act. Sev. 23, II5?

complur[ Act. Aug. 52

componere Act. Aug. I49; Act. Sev. 235

concurrere Act. Sev. 256

condere Act. Sev. I3, 24, 245

conferre Act. Aug. I I, 69; Act. Sev. 10, 24, 258 
conficere Act. Aug. I00

coniux Act. Aug. 66; Act. Sev. I 52, 178 conlega ( $\rightarrow$ collega) Act. Aug. $\mathrm{AB}_{3}$; Act.

Sev. 60, 6I, 62, 63

connexus Act. Sev. 235, $25 \mathrm{I}$

conscriptus Act. Sev. 22

consecrare Act. Sev. 47

consedere Act. Sev. 254, 268

consequi Act. Sev. 258

conservare Act. Aug. 59

consistere Act. Sev. 6

constituere Act. Aug. $\mathrm{Ab}_{3}, \mathrm{Ab}_{4}, 76, \mathrm{I}$, 8;

Act. Sev. I55, 223, $229 f$.

consul Act. Aug. AB I, Ab4, AB 6, C 3,29 ,

37, 52, 59, 6I; Act. Sev. 6, I4, 67, 68, 8I,

90, I44, 207, 255, 270

consulere Act. Sev. 62

consummare Act. Sev. 208, 253, 253 f., $266 f$.

contaminatio Act. Sev. Iogf.

continuus Act. Sev. 30

contio Act. Aug. 26, 27

convenire Act. Aug. I5, I8, 36, 79; Act.

Sev. $28,53,60,64,75(2), 25 \mathrm{I}$

corbis Act. Sev. 99

cornicen Act. Sev. 247

corona Act. Sev. 165, I66, I69, I79, I80, 234

coronatus Act. Sev. 223, 229, 235

corrigere Act. Sev. 36

cotorius Act. Sev. I8I, 225

culter Act. Sev. I65, I8I, 225

cultus Act. Aug. II3; Act. Sev. 9, 23

cupere Act. Sev. 2 I 8

cura Act. Sev. 7, 8, I0

curare Act. Aug. Ab $5, \mathrm{C}_{4}, 6_{3}$; Act. Sev. 22

curator Act. Sev. 67, 208, 269

cursor Act. Sev. $215,248,255$

custos Act. Sev. 2 I 8

Cymaeus Act. Sev. 236

d.e.r.i.c. Act. Aug. $\mathrm{AB}_{2}, \mathrm{C}_{2}, 55,6 \mathrm{I}$

dare Act. Aug. AB 5, $\mathrm{C}_{4}, 30,63,95, \mathrm{I} 28$,

I43, I63; Act. Sev. 80, I 30, I66, I 86, 2 I4,

$215,217,252,258,259$

dea Act. Aug. Cin , 90, in

debere Act. Sev. 61, 63, 64

decem Act. Sev. 28, 43

decernere Act. Aug. AB I; Act. Sev. 23

decimus Act. Aug. Cr6, 25; Act. Sev. 20

decretum Act. Sev. 29 dein Act. Sev. 183, 214, 224, 230

deinde Act. Aug. 36, I08, I23; Act. Sev. I67

demum Act. Aug. C I4

denuntiare Act. Aug. I5, 78, I0I, II4, I23, I47; Act. Sev. I 84, 235

denuo Act. Sev. 7

descendere Act. Sev. 80

designatus Act. Sev. 67(2), 68, 8I, 90, I44, 207(2), 255, 270, 271, 300

desultor Act. Aug. I 54; Act. Sev. 2 I 5, 216 , $248,255,258,258 f$.

deus Act. Aug. Ci I, I I, II 3; Act. Sev. 9, I 2,24

devotio Act. Sev. I9

dexter Act. Sev. I 59, I8I, 224, 246

dicere Act. Aug. 24, 46, 64, I IO, I55, I62; Act. Sev. 74, II 4, 2I 2, 25 I, 279

dies Act. Aug. AB 6, Ab8, 4, 5, I6(2), 24, $37,4 \mathrm{I}(2), 48(2), 50,55,58,64,67,77,82$, 83, I I9, I 39, I 56, I 59, I60, I62; Act. Sev. $28,29,45,59,62,64,71,75,77,79,179$, 209, 2I $2,2 \mathrm{I} 3,2 \mathrm{I} 4,2 \mathrm{I} 9,220,22 \mathrm{I}(4), 222$, $236,259,273$

diligens Act. Sev. I3

diligenter Act. Aug. 7, I3, 23, I I 3; Act. Sev. 79

dimittere Act. Aug. I 53

discrimen Act. Sev. 235

dissignator Act. Sev. 280

distribuere Act. Sev. 6I, 64, I I 7

dividere Act. Sev. 82, 83, 84, I68, 252

domi Act. Aug. $\mathrm{C}_{13}$

dominus Act. Sev. 78

domus Act. Aug. 97, 99, I 30 ; Act. Sev. I3 I, I 36, I 39, I42, I64, I66, I 87

ducere Act. Sev. 7I, 280

duelli domique Act. Aug. 94, I27; Act. Sev. I 29, I 39, I 58, I73, I 82, I 86, 226f., 232 duo Act. Aug. 102; Act. Sev. I 57

dux Act. Sev. 246

eborneus Act. Sev. 91, 254, 288, 302

edere Act. Aug. 57; Act. Sev. I, I 5 ?, 28, 62, $96, \mathrm{IO} 3,2 \mathrm{I} 3$

edicere Act. Aug. 40; Act. Sev. Is ?, 77

edictum Act. Aug. 24, 46, 64, 77, IIO, I I4, I55, I62; Act. Sev. 7I, II I , 209, 273

Eidus $\rightarrow$ Idus

epistula Act. Sev. 59 
epulari Act. Sev. I 83

eques Act. Sev. 317

equestris Act. Sev. 80, 98f., 202

ergo Act. Aug. 92, 98, 105, I17, I21, I36,

I4I, I43; Act. Sev. 2I, I28, I33, I38, I4I,

I 57, I63, I72, I 82, 226, 23 I

eventus Act. Sev. 218

exemplum Act. Aug. 26, I I I

exhibere Act. Sev. 214, 2 I7

existimare Act. Sev. 77

existumare Act. Aug. 65

expensum Act. Sev. 23

exuviae Act. Sev. 304

facere Act. Aug. $\mathrm{AB}_{\mathrm{I}}, \mathrm{AB}_{4}, \mathrm{AB} 6,38,5 \mathrm{I}$, 52, 59, 96, I I 5, I 29, I 33, I39, I 53; Act.

Sev. I6(2), 26, 28, I 30 of., I 80, I 87,224 , 230,284

factio Act. Sev. 248, 255

familia Act. Aug. 97, 99, I30; Act. Sev. I3 I, I 36, I 39, I 42, I64, I66, I 87

fari Act. Sev. 242

fas Act. Sev. Io8

faustus Act. Sev. I04

favere Act. Aug. 95, I 28; Act. Sev. I30, I 86

felicitas Act. Sev. I I, 20, 26, 30

felix Act. Sev. I9, I04

femina Act. Aug. 73, I I4; Act. Sev. 30

fera Act. Sev. 2 I 8

feriae Act. Aug. 39; Act. Sev. I 5, 29, 44, 78

ferre Act. Sev. 304

festin[ Act. Sev. Is

festus Act. Sev. 7

fetasius Act. Sev. 257

fieri Act. Aug. $\mathrm{AB}$ 2, 93, І06, I I0, I I 8, I 22,

I37, I42; Act. Sev. 8, 23, 28, 29, 42, 53,

I $35, \mathrm{I} 42, \mathrm{I} 57, \mathrm{I} 63, \mathrm{I} 72, \mathrm{I} 82,226,232$

filius Act. Sev. 2I, 55, 57, 71, 73, I I 2, I I4, I $80,209,2$ II 224, 228, 230, 274, 277, 317

fimbriata Act. Sev. I 56, 224, 227

findere Act. Sev. 244

fito Act. Aug. 99, I44; Act. Sev. I66

frater Act. Sev. 55, 72, I I 2, 209, 274

fraus Act. Aug. 55, 57

frequens Act. Aug. IO, 2 I, I I I; Act. Sev. 249

frequentare Act. Sev. I I, 24

frons Act. Sev. I65

fructus Act. Sev. I I, 76 fruges Act. Aug. I I, 30, 32, 35, 82, 89; Act. Sev. I 2, 100, 258, 259(2)

fundere Act. Sev. 243

funus Act. Aug. 88

futurus Act. Aug. 53, 6I(2), 62; Act. Sev. 2I, 22, 3I

gaudium Act. Sev. 2 I

generatim Act. Aug. 69

genitalis Act. Sev. 22

gens Act. Sev. 237

genu Act. Aug. I26, I 3 I; Act. Sev. I85, I 87

genus Act. Sev. 2 I

glaucus Act. Sev. 244

Graecus Act. Sev. I 81, 225, 267

Graecus asticus Act. Aug. I57, I6I

Graecus thymelicus Act. Aug. I57, I6I

gratia Act. Sev. 24

grex Act. Sev. 238

habere Act. Aug. 2I, IOI, I09, I38; Act.

Sev. 24, 68, 95, 206, 228, 25I, 259

habitare Act. Sev. 78

harum rerum Act. Aug. 98; Act. Sev. I33, I 4 I

haruspicatio Act. Sev. I64

homo Act. Aug. 65

honor Act. Aug. I I3; Act. Sev. 237

honorarius Act. Aug. I 56, I60; Act. Sev. 206, 209, 2I 2, 2I9, 230, 28 I

honorare Act. Sev. $25 \mathrm{I}$

hora Act. Aug. 81, 84, I53, I57(2), I58

hortari Act. Sev. 218

hostia Act. Sev. I65

hs (sestertius) Act. Sev. 2 I4(2), 2 I $5(4)$, $2 \mathrm{I} 6(4), 2 \mathrm{I} 7(4)$

humanus Act. Sev. 20, 22

ibi Act. Sev. 81, 82, I79, I83, I84, 209, 223, 229, 234, 267(2), 268

ibidem Act. Aug. 58, 103

ideo Act. Sev. 2 I

Idus Act. Aug. I62, I63, I64; Act. Sev. 49, 2I 3, 2I 4, 22I, 279

imminere Act. Sev. 22

immolare ( $\rightarrow$ inmolare) Act. Sev. I4I(2), I60, I65, I69, I 8 I, 225, 260, 287

immortalis ( $\rightarrow$ inmortalis) Act. Sev. 9, 23, 24

impartire Act. Sev. I0, I68(2) 
imperare $\rightarrow$ inperare

imperium Act. Aug. 93, I 26; Act. Sev. 37, I07, I I I, I 28f., I 39, I 57 f., I73, I 82, I86, 226, 232, 242

impleare Act. Sev. 9

incipere Act. Aug. I09

inde Act. Sev. 97, 100, 252, 260

infra Act. Aug. AB 7; Act. Sev. 260

inlex Act. Sev. 237

inluciscere Act. Aug. 4, 4I

inmolare ( $\rightarrow$ immolare) Act. Aug. 90, 98, 99, I03, II9, I 34

inmortalis ( $\rightarrow$ immortalis) Act. Aug. C I I

inperare Act. Aug. $\mathrm{AB}_{5}, \mathrm{C}_{4}, 6_{3}$

inquit Act. Sev. 20

inscribere Act. Aug. 60, 62

insequi Act. Aug. 90

inspicere Act. Sev. 68

instituere Act. Aug. 53, 56; Act. Sev. 8, I4

integer Act. Sev. I68

intercedere Act. Sev. 249

interesse Act. Aug. 54; Act. Sev. 209, 228

intermittere Act. Aug. 109, I 59

invenire Act. Aug. $\mathrm{AB}_{2}$

item Act. Aug. 22, 35, 43, 85, 86; Act. Sev. 206, 213, 220, 22I, 222, 248, 252, 255 , 260

iubere Act. Aug. 6, 8; Act. Sev. 23, I6 I

Iunius Act. Aug. 4, 5, 7, 16(3), 41, 42, 49, 50, 64, 67, 77, 82, 84, 89, I03, I I9, I 39, I 56, I 59, I60, I62, I63, I64; Act. Sev. 60, 64, 79, I79, 212, 219, 22 I(2), 228, 266, 279

ius Act. Sev. 77, 108

iussu Act. Sev. Ios

iustus Act. Aug. I I I

iuxta Act. Aug. I53

Kalendae Act. Aug. AB 6, 4, 16, 37, 4I, 48, 49, 50, 64, 67, 77, 82, 84, 89, I03; Act. Sev. $59,60,64,79$

labi Act. Sev. 7

laetitia Act. Aug. I I I; Act. Sev. 21, 26, 76 laevus Act. Sev. I 8I,225

lanx Act. Sev. 252

Latinus Act. Aug. 83, 85, 94, I08, I 27, I 56, I60; Act. Sev. I29, I39, I 58, I64, I73, I $82, I 86,227,232,239,267$

lauriger Act. Sev. 237 laurus Act. Sev. I 20

lea Act. Sev. 2 I 9

legere Act. Sev. 6, 25, 61, 63, 23I

legio Act. Aug. 95, I 28; Act. Sev. I30, I 86

leo Act. Sev. 2 I 9

leopardus Act. Sev. 219

lex de marit. ordin. Act. Aug. 57

libare Act. Aug. I43

libellum Act. Sev. 6

libenter Act. Sev. 95

liber Act. Aug. AB 2, 92, I05, I I7, I 2 I, I 36 , I4I; Act. Sev. I28, I38, I57, I63, I72, I 8 I, 226, 23 I

liberi Act. Aug. 8, 65

libum Act. Aug. II5, II 8, I39, I42, I43;

Act. Sev. 172, 231, 232

licere Act. Aug. 56, 57

ligneus Act. Aug. I08, I 57, I6r; Act. Sev. I 55, 208, 2I 3, 220, 22 I, 222, 223, 229, $253,267,269$

litis Act. Aug. 13

loca Act. Sev. 280

locare Act. Aug. $\mathrm{AB}_{3}, \mathrm{AB}_{7}, 6_{3}$

loce[ Act. Aug. 77

locus Act. Aug. 61, 62, I 53; Act. Sev. 24, 61, 64, 82, 108

loedi Act. Aug. 39

longus Act. Sev. 20

lucar Act. Aug. $\mathrm{AB}_{\mathrm{I}}, \mathrm{AB}_{4}$

luctus Act. Aug. I I 2, I I 4; Act. Sev. 30

ludere Act. Sev. 252

ludio Act. Sev. 248

loedi Act. Aug. 39

ludus Act. Aug. $\mathrm{AB}_{1}, \mathrm{AB}_{3}, \mathrm{AB}_{4}, \mathrm{AB}_{9}$, $\mathrm{C}_{2}, \mathrm{C}_{17}, 22,25,52,53,54$ f., 55, 56, 59, 6I, 62,(2), 68, 83, 85, 100, I08, I09, I I 2, I33, I 53, I 56(2), I60(2); Act. Sev. I, 7, I3, I 7, 2 I (2), 26, 27, 29(2), 31 , 47, 62, 74, 76, 103, 178, 206, 208, 209(2), 21 2(2), 219, $228,233,253(2), 266,267(3), 268,28$ I

lustrare Act. Sev. I02(2), I06, I09

lusus Troiae Act. Sev. 252

machina Act. Sev. 2 I 8

macte Act. Aug. 98, I43; Act. Sev. I33, I4 I magister Act. Aug. 57; Act. Sev. 6, I4, 67, 88, I0I, 207

magistratus Act. Sev. 98

maiestas Act. Aug. 93, I27; Act. Sev. I29, I 39, I 58, I 73, I 82, I $86,226,232$ 
maior Act. Aug. 96, I 29; Act. Sev. I 30, I 87 maiores Act. Aug. 17, 26; Act. Sev. 24

Maius Act. Sev. 59

mandatus Act. Sev. ios

manuarium Act. Sev. 25

manus Act. Sev. I80, 218 , 224(2), 235, 25 I maritatus Act. Aug. 55

marmor Act. Sev. 30

marmoreus Act. Aug. 60, 62

Martius Act. Aug. AB 6; Act. Sev. I I

mas Act. Sev. I 57, I63

mater Act. Aug. C9

mater familiae/-as Act. Aug. 80, I09, I23, I 25, I 30; Act. Sev. I 85 , I 87

matrimus Act. Aug. 20, I47; Act. Sev. 235

matrona Act. Aug. 78, IoI, I I2, I 38 ; Act.

Sev. 63, I78, I 84, I $88,206,228,259,260$

maximus Act. Sev. Io

medius Act. Sev. 305

melius Act. Aug. 92, 105, I17, I21, I25,

I36, I4I; Act. Sev. 108, I 28, I38(2), I 57 ,

I63, I72, I 82, I $85,226,23 \mathrm{I}$

meminisse Act. Aug. I3, 23; Act. Sev. I0

memoria Act. Aug. 59, 6I, I I3; Act. Sev. 20,30

meridies Act. Sev. 216

merito Act. Sev. 9

merx Act. Sev. 78

meta Act. Aug. I 54; Act. Sev. 255

miles Act. Sev. $78 \mathrm{f}$

milicheus Act. Aug. I I

militia Act. Aug. $\mathrm{C}_{13}$

minor Act. Aug. 47

minuere Act. Aug. I I2, I I 4

missus Act. Sev. 214, $215(3), 216(2), 255(3)$, $257(3), 258$

mitis Act. Sev. 238

mittere Act. Aug. I 54(2), I65; Act. Sev. 59, 68, 2I 5,2 I6(2), $255(2), 257(2), 258$

modus Act. Aug. 82, 9I(2), I09, II6, I20, I35, I48; Act. Sev. 227, 233

molestia Act. Aug. 47

monitus Act. Sev. 8

mortalis Act. Aug. 56

mos Act. Aug. 26, I I I; Act. Sev. 206, 256

mox Act. Sev. I64

mulcere Act. Sev. 237

mulier Act. Aug. 7 I

munificentia Act. Sev. 219

Murcius Act. Sev. 255 natus Act. Aug. I7; Act. Sev. 238

nemo Act. Aug. 54

nepos Act. Sev. 55, 57, 72, 73, I I 2, I I4, 2 IO, 2 I I, 278

Neptunius Act. Sev. 244

nixus Act. Aug. I26, I3 I; Act. Sev. I85, I 87

noctu Act. Aug. I6, I34

nocturnus Act. Sev. 228

nomen Act. Aug. AB 4; Act. Sev. 30, 77, 260

Nonae Act. Aug. 5, I6(2), 42, I I9, I 39, I 56, I59, I60; Act. Sev. I79, 2 I 2(2), 2 I 3, 2 I9, $221,228,266$

noscere Act. Sev. 242

novem Act. Sev. 172, 232(3), 259

November Act. Sev. 49

noveni Act. Sev. 23I(3)

novus Act. Sev. 220(2), 235

nox Act. Aug. 4, 40, 84, 90, I00, I09, I I 5 , I53; Act. Sev. 62, 75, 78, 222, 227, 233, 236

nullus Act. Aug. I00

numen Act. Sev. 17, 237

numerus Act. Sev. I9

nungentesimus Act. Sev. I 3

nuper Act. Aug. $\mathrm{AB}_{3}$; Act. Sev. 239

nupta Act. Aug. I23, I26, I3 I; Act. Sev. I 85,187

obesse Act. Sev. 30

obliquus Act. Sev. I8I, 225

obsecrare Act. Aug. I26, I3 I; Act. Sev. I 85 f., i 88

observare Act. Aug. I I 3

obtemperare Act. Aug. 94, I 27; Act. Sev. I 29, I39, I 58, I64, I73, I 82, I 86, 227,

232

occupare Act. Sev. 45

odium Act. Sev. 2 I 3

odor Act. Sev. 63

officium Act. Aug. I 44

omnis Act. Aug. C I I, 8, 36, 65, 87, I66;

Act. Sev. 23, 24, 43, I09, I 37, 252

onager Act. Sev. 219

oportere Act. Aug. C I, 72; Act. Sev. 62

optimus Act. Sev. 17, 26, I07, I09

opus Act. Aug. AB 6

ora Act. Sev. 242

orare Act. Aug. I 26, I3 I; Act. Sev. I 8 5, I 88 
ordo Act. Sev. 80f., 99, II 5, 214, 219, 227, 233

origo Act. Sev. 7, is

otium Act. Sev. 3 I

palam Act. Aug. 26

palliolatus Act. Sev. 235

palmatus Act. Sev. 254

pantomimus Act. Sev. 220, 220f., $22 \mathrm{I}(2)$, 222(2)

par Act. Sev. 217

parare Act. Aug. 22; Act. Sev. 2 I 8

patefacere Act. Sev. I6

patera Act. Sev. I8 I, 225

patrare Act. Sev. 8

patres Act. Sev. 7, 22

patrimus Act. Aug. 20, I47; Act. Sev. 235

pecunia Act. Aug. $\mathrm{C}_{2}, \mathrm{C}_{4}, \mathrm{AB}_{5}, 6_{3}$; Act.

Sev. 48

peragere Act. Aug. 166; Act. Sev. 2 I 2

percipere Act. Sev. 217

perficere Act. Aug. I47; Act. Sev. 217, 227, 233, $25 \mathrm{I}$

perpetuus Act. Sev. 259

perspectare Act. Sev. I78, 268

perspicere Act. Sev. 234

pertinere Act. Aug. 59, II 3

pervenire Act. Sev. 76

pervigil Act. Sev. 8

pervigilium Act. Sev. I 5 of.

petere Act. Aug. 8, 66; Act. Sev. 26, I68, 256

Phoebeius Act. Sev. 236

piare Act. Sev. 102, 106, I09

pilus Act. Sev. I65

pius Act. Sev. 19, 26, 27, 94

placere Act. Aug. I I 2; Act. Sev. 76

plebs Act. Sev. 8I, 9I, IOI

plebs Romana Act. Aug. $\mathrm{C}_{13}$

plenus Act. Aug. I34, I37; Act. Sev. 225, 226

polliceri Act. Sev. 217

pollicitatio Act. Sev. 216

pompa Act. Aug. 164; Act. Sev. 248, $250(2), 253$

ponere Act. Aug. 75, I00, 102, I54(2); Act.

Sev. 99, I 80, 224, 227

pontifex maximus Act. Sev. 2

popanum Act. Aug. II5, II8, I40, I42,

I43; Act. Sev. 172, 23 I, 232

populus Act. Sev. 61, 64, 82, 83, 90f., Ioof., 249

populus Romanus Act. Aug. $\mathrm{C}_{13}, \mathrm{C}_{14}, 92$, 93, 95(3), 96(2), 99, I05, I I7, I 2 I , I 25 (2), I 27, I 28(3), I 29, I30, I 36, I4I; Act. Sev. 29, 8 I, I I 5f., I 28, I29, I30(4), I3 I, I36, I38, I39, I42, I57, I 58, I63(2), I66, I72, I73, I 82(2), I85(2), I86(3), I87(4), 226(2), 232(2), 258

porcilia Act. Sev. 260

porcus Act. Sev. 279, 285,286

porro Act. Aug. C I 2

porticus Act. Aug. 32, 33; Act. Sev. I 19

poscere Act. Sev. 22

posse Act. Sev. 8, I06

postis Act. Sev. 245

postmeridianus Act. Sev. 257

postquam Act. Sev. 217

praecedere Act. Sev. 250, 260

praeesse Act. Aug. AB 5, C 3,63

praeferre Act. Aug. I64

praeire Act. Aug. I 24; Act. Sev. 93, 165 , I $80,185,224,230$

praemium Act. Sev. 215, 258

praesens Act. Sev. 22

praesessio Act. Sev. 206

praeside[ Act. Sev. 28I

praetexta Act. Sev. 179, I80, I83, 224, 227

praetextatus Act. Sev. 82, I I7f., 223, 229, $235,252,266$

praetor Act. Aug. $\mathrm{AB}_{4}, \mathrm{C}_{3}, 6_{3}$

praetorius Act. Sev. 268

precari Act. Aug. 91, 93, I04, I I6, I I 8, I 20, I 22, I 26, I3 I, I 35, I37, I40, I42, I43;

Act. Sev. I 28, I 38, I $57(2)$, I62f., I63, I70, I73, I 82,185, I $88,226,232$

precatio Act. Sev. I 56f, I 8 I (2), $225(2), 23$ I prex Act. Aug. I 43

pridie Act. Aug. 49, 89, I33, I38, I59, I63, I64; Act. Sev. 5, 21 2, 219,266

primitiae Act. Aug. I I

primum Act. Sev. I I 8

primus Act. Sev. 156, 214, 217, 219, 220, 227, 24 I, 255

princeps Act. Sev. 10, 26, 27

principalis Act. Sev. 7

prius Act. Sev. 52

procedere Act. Sev. 97, 209, 229, 253, 267

procurare Act. Sev. 96 
prodigivus Act. Aug. 9I(2), I34; Act. Sev. 225,226

proinde Act. Aug. I I I

pronaus Act. Sev. 234, $25 \mathrm{I}$

pronepos Act. Sev. 55, 57, 72, 74, I I 2, I I4, 210, 2 II, 278

propago Act. Sev. 17

propitius Act. Aug. 96, 99, I 29, I44; Act.

Sev. I3 I, I $35 f .$, I 38, I 42, I63, I66, I 87

proponere Act. Aug. 24, 46, 64, IIO, I55,

I62; Act. Sev. 7I, I I I, 209, 2 I9, 273, 280

proprius Act. Aug. 93, 98, I03, I I9, I34,

I37; Act. Sev. I38, I4 I, 226

propter Act. Aug. 54

prosper Act. Sev. 22

protegere Act. Sev. 246

prout Act. Sev. I 54

providentia Act. Sev. 7, I I

providere Act. Sev. 8, 218(2)

proximus Act. Aug. AB I; Act. Sev. 60, 279

pthois Act. Aug. II5, II 8, I40, I42, I45;

Act. Sev. 172, 231, 232

publicus Act. Aug. I I I; Act. Sev. 8, 76

publicus (=servus) Act. Sev. 167, 224, 249?

pudens Act. Sev. 240

puella Act. Aug. I47; Act. Sev. 235(2), 249, 250, 25I, 252, 260, 263

puer Act. Aug. 20, 73, I47, I64; Act. Sev.

I $8,234,235,238,249,250,25 \mathrm{I}, 252,260$, $29 \mathrm{I}$

pulcher Act. Aug. 106, I22; Act. Sev. 94, I $57, \mathrm{I} 60,163,165,169, \mathrm{I} 82$

pulvinar Act. Sev. 283, 284, 304, 305

purgamentum Act. Aug. 30

purgare Act. Sev. 106, I09

purificare Act. Sev. 224

q.d.e.r.f.p. Act. Aug. C If., $\mathrm{AB}$ 2, 55, 6I

quadriga Act. Aug. I 54, I65; Act. Sev. 214, $215,217,248,255,258(2)$

quaerere Act. Sev. I57, I63, I73, I82, 226, 232

quaesere Act. Aug. 93, I06, I 18, I 22, I37, I 42 ; Act. Sev. I 28, I 38

quaestor Act. Sev. 67, 68, 87, 207, 208, 269, 27I, 300

quartus Act. Sev. 2Is

quarum rerum Act. Aug. 92, 105, I I7, I 2 I, I36, I4I; Act. Sev. I 28, I38, I 57, I63, I72, 226, I 8 If. quietus Act. Sev. 32

quinquagensimus Act. Sev. I3

quintus Act. Sev. 216

Quirites Act. Aug. C I4, 92, 95(2), 96(2), 97, 99, I06, I17, I 21, I 25, I 27, I 28(2), I29(2), I30, I3I, I36, I4I; Act. Sev. 78, I 28, I 29, I 30(4), I 3 I, I36, I 38, I 39(2), I42, I 57, I 58, I63(2), I66, I72, I73, I $82(2)$, I $85(2)$, I $86(3)$, I $87(4), 226(2)$, $232(2)$

ratio Act. Sev. 22, 78

se recipere Act. Sev. 252

recte Act. Sev. 8

rector Act. Sev. 20

reddere Act. Sev. 170, 236

redemptor Act. Aug. 63

referre Act. Aug. 29, I I 2; Act. Sev. 24

regio Act. Sev. 79

religio Act. Aug. 54, 56, 59, 6r; Act. Sev.

30, 91, I 83

relinquere Act. Sev. 8I

reliquus Act. Sev. 252, 259

renovare Act. Sev. 256

repetere Act. Sev. 46, 77

res Act. Aug. C 3, 38, 43; Act. Sev. 48

res divina Act. Aug. I 8; Act. Sev. 106, I80, 230

res publica Act. Aug. 96, I 29; Act. Sev. 96, I 30, I 87

retenere Act. Sev. 225

retinere Act. Sev. I 80, I8I, 225

reus Act. Sev. 30, 46

ripa Act. Sev. I 20

ritus Act. Aug. 2, 9I, II9, I34; Act. Sev. I09, I 8I, 225

Romanus Act. Sev. 22, 37, 317

sacerdos Act. Aug. $\mathrm{AB}_{3}, \mathrm{AB}_{7}, \mathrm{I}_{5}, 22,25$, 97, I00, II 5, I39, I47, I 53; Act. Sev. 19, 43

sacrificalis Act. Sev. 253

sacrificare Act. Sev. I83, 23 I, 233

sacrificium Act. Sev. 29, I3 I, I34, I4I, I 59, $227(2), 233(2), 25 \mathrm{I}$

sacrum Act. Aug. 86, 93, I06, I I 2, I I 8, I 22, I37, I42; Act. Sev. 9, 23, I02, I 28, I38,

I $57,163,172, I 82,226,232$

saecu[ Act. Sev. I 8

saecularis Act. Aug. $\mathrm{AB}_{\mathrm{I}} \mathrm{AB}_{3}, \mathrm{AB}_{7}, \mathrm{AB}_{9}$, 
$\mathrm{C}_{17}, 25,52,53,60,83$; Act. Sev. I, 8, I2, hs (sestertius) Act. Sev. 214(2), $215(4)$, I 4, I 7, 2I, 26, 27, 47, 62, 74, 76, I02, $253(2)$

saeculum Act. Sev. 7, I0, 16, 22 salus Act. Aug. $\mathrm{AB}_{4}$; Act. Sev. 60, 62 salutaris Act. Sev. I०4

salvus Act. Aug. 96, I 29; Act. Sev. I30, I 87 sanctus Act. Sev. 24, 26, 27

satis Act. Aug. 27

scaena Act. Aug. I00, I 54; Act. Sev. 178, 233

scaenicus Act. Aug. I 53; Act. Sev. 21 3, 267 scipio Act. Sev. 91, 254, 302

scribere Act. Aug. $\mathrm{AB}_{7}, \mathrm{AB} 8,50,58,92$, I05, I 17, I2 I, I36, I4I; Act. Sev. I 28, I 38, I 57, I63, I72, I 8 I, 226, 23 I secare Act. Sev. I65 secretus Act. Sev. 245 sectari Act. Sev. 2 I 9 secundum Act. Aug. 108 secundus Act. Sev. II8, I62, 214, 2 I5(2), $2 \mathrm{I} 6,2 \mathrm{I} 7(2), 22 \mathrm{I}(2), 233,258,259$

securitas Act. Sev. 23, 2 I 8

sedere Act. Sev. 257

sedile Act. Aug. Io I

sedis Act. Sev. 268

sella Act. Aug. I02

sellis Act. Sev. 240

sellisternia Act. Aug. 39, 7I, IOI, I09, I38;

Act. Sev. I79, 205, 228, 260

semel Act. Aug. 56, 66

semper Act. Aug. 94, I27; Act. Sev. I 29,

I 39, I 58, I64, I73, I 82, I 86, 227, 232

sempiternus Act. Aug. 94f., I 28; Act. Sev. I 29, I 86

senator Act. Sev. 44

senatoris Act. Sev. 260

senatus consultum Act. Aug. AB 6, 51, 58

separatim Act. Aug. 74

septimus Act. Sev. I, I7, 74, 217

septingenti Act. Sev. 2 I 8

sequens Act. Aug. AB 2, AB 6

sequi Act. Sev. 28, 227, 233

sericus Act. Sev. 252

serva+[ Act. Sev. 33

servare Act. Aug. 96, I 29; Act. Sev. 29, 44, I 30, I 87,2 I 6

sescentensimus Act. Sev. Is

sexagensimus Act. Sev. Is

sextus Act. Sev. I3, 2 I6

$$
2 \mathrm{I} 6(4), 2 \mathrm{I} 7(4)
$$

signare Act. Sev. 236

similis Act. Sev. 256

similiter Act. Sev. 233

singuli Act. Aug. 34; Act. Sev. 214, 248, 255,257

solea Act. Sev. 257

solere Act. Aug. AB 2; Act. Sev. 28, 42

sollemnis Act. Aug. 8I, I I 2, I56, I60; Act.

Sev. 9, 23, 45, 75, 2I 2, 252

sors Act. Sev. 68, 7I

sortiri Act. Sev. 61, 64, 82, 206

spargere Act. Sev. I78, 234

spectaculum Act. Aug. 54; Act. Sev. 213, 214,256

spectare Act. Aug. 56, 57

spes Act. Sev. 22

spirare Act. Sev. 245

splanchna Act. Sev. I70, I7I

stare Act. Sev. 268

statuere Act. Aug. I I3; Act. Sev. 63

sternere Act. Aug. 72

struthio Act. Sev. 2 I 9

subscribere Act. Sev. 260

subsequi Act. Sev. 250

subsidium Act. Sev. I 8

suburbanus Act. Sev. 78

suffimenta Act. Aug. 48, 76; Act. Sev. 6I, $64,80,82,83,84$

suffitus Act. Aug. 68

suggestum Act. Sev. 5

sumere Act. Sev. I 56, I79, I 83, 224, 227,

234, 254

summa Act. Aug. $\mathrm{AB}_{2}, \mathrm{AB}_{3}$

sumptus Act. Sev. 23, 29

superbus Act. Sev. 238

superior Act. Aug. 89, I 53

superius Act. Sev. I6

supplicare Act. Sev. 63, I 88

supra Act. Aug. I06, I I 8, I 22, I 37, I42;

Act. Sev. I 39, I 58, I64, I73, I 82, 227 ,

232, $254,257,28$ I

sus Act. Aug. I34, I37; Act. Sev. 225, 226

suscipere Act. Sev. I67

sustinere Act. Sev. 250

tantus Act. Aug. 59, 6I; Act. Sev. 30

tegere Act. Sev. 250

tempestas Act. Sev. I 2 
temporalis Act. Sev. I55, 223, 229, 253, 254 tempus Act. Aug. II 2; Act. Sev. 7, I 5, 16, I $8,20,22,25,26,52$

tenere Act. Aug. 57; Act. Sev. 20

terni Act. Aug. 36; Act. Sev. 167

terra Act. Sev. 244

tertius Act. Sev. 215(3), 216, 217(2), 22I, 222, 223

tessera Act. Sev. 68, 206

tetrastylum Act. Sev. 230

theatrum Act. Aug. 100, 108, I 54, I 56, I 57, I6I(2); Act. Sev. I45, I78, 208, 2 I3, 220(2), 22I, 222, 233, 253, 267(2), 268, 27I, $28 \mathrm{I}$

tibicen Act. Sev. 236, 247

tiro Act. Sev. 248

togatus Act. Sev. 247

tot Act. Sev. 22

totidem Act. Aug. I47; Act. Sev. 235

se transferre Act. Sev. 267

tralatus Act. Sev. 250

translatus Act. Sev. 247

tres Act. Sev. 75, 213, 252

tribuere Act. Sev. I I

tribunal Act. Aug. 69; Act. Sev. 6I, 64, 68, $69,80,82,97(2)$, I00, I I 7, 253, 268

tribunicia potestas Act. Aug. 53; Act. Sev. $3,56,60,72,73$, II 3, 210, 2 I I , 276, 277 tribus Act. Aug. AB 8, Abg(2)

tricensimus Act. Sev. 77

triduum Act. Sev. 30

tubicen Act. Sev. 247

tunica Act. Sev. I 56, 224, 227

turma Act. Sev. 24I

tus Act. Sev. 180, 224, 230

tutela Act. Sev. 79

ubertas Act. Sev. Io

ubique Act. Sev. 2 I 8

una cum Act. Sev. 78 universus Act. Sev. 76

urbanus Act. Sev. 78

urbs Act. Sev. I3, I4, 242

urna Act. Sev. 68, 206

ursus Act. Sev. 2 I 9

vacca Act. Sev. I 8 I, 285

valere Act. Sev. 61, 63

valetudo Act. Aug. 95, I28; Act. Sev. I 29f., I 86

vatis Act. Sev. 8

velle Act. Aug. 87; Act. Sev. 219, 280

velum Act. Sev. 252

venatio Act. Aug. I63; Act. Sev. 2 I 8

veneratio Act. Sev. 23

venire Act. Aug. 68; Act. Sev. 179, I84, 223, 254, 267

verbum Act. Aug. $\mathrm{AB}$ I, $\mathrm{AB}_{7}, 24,46,52$, 59, I0I, I24, I46, I 55, I62; Act. Sev. 26, 59, 7I, 93, III, I 50, I 53, I68, I 85,209 , $233,273,284$

verruncare Act. Aug. C I4

Vestalis Act. Sev. I 2 I, I 85

vetus Act. Sev. 7

vetustus Act. Sev. 8, I 4

victoria Act. Aug. 95, I 28; Act. Sev. I29, I 86

victrix Act. Sev. 245

videre Act. Sev. 26

videri Act. Aug. 38, 43, I I3; Act. Sev. 60

vincere Act. Sev. 214, 215, 216, 2 I6f.

vindiciae Act. Sev. I I

vinum Act. Sev. I 80, I 8 I , 224, 225, 230

vir Act. Sev. 26, 29

virgo Act. Aug. 20; Act. Sev. I 2 I, I 85

vita Act. Sev. 20

volens Act. Aug. 96, 99, I 29, I 44; Act. Sev. I3 I, I 35, I3 8, I 42, I63, I66, I 87

votum Act. Sev. 238

vox Act. Sev. I9, 24I 
Index nominum et locorum Act. Aug.

\section{Augustus \\ Imperator Caesar Augustus C 5, 52f., 90, I03, I I f., I 34f., I66; Imperator Augus- tus I39; Imperator Caesar I 50 ; magister XVvirorum s.f. C 5; Caesar I07; tribu- nicia potestas 53}

\section{Agrippa}

M. Agrippa 44, 53, I04, I07, I 20, I 23, I32, I39, I 50, I65, I66; tribunicia potestas 53

\section{Iulius Caesar \\ Caesar $\mathrm{AB}_{4}$}

\section{Quindecimviri}

XVviri s. f. $\mathrm{AB}_{3}, \mathrm{I}, 24,29,46,57,64,97$, 99, I0I, IIO, I30, I50, I55, I62, I66; magistri XVvirorum s. f. 29, 57

Q. Aelius Tubero $\mathrm{C}_{7}$, I 52, I68

Q. Aemilius Lepidus 50f., I 50, I 54, I66

L. Arruntius C7, 45, I5 I, I67

C. Asinius Gallus AB 9, C7, I07, I5 I, I68

C. Caninius Rebilus $\mathrm{C}_{7}$, I07, I52, I68

M. Claudius Marcellus C7, I5 I, I68

M. Cocceius Nerva C6, I 5 I, I67

M. Fufius Strigo $\mathrm{C}_{7}, \mathrm{I} 5 \mathrm{I}, \mathrm{I} 67$

D. Laelius Balbus $\mathrm{C}_{7}$, I5 I, I68

C. Licinius Stolo I 50, 167

M. Lollius 45, I07, I I I, I67

L. Marcius Censorinus C 5, 44, 166

C. Mucius Scaevola I07, I 50, I67

C. Norbanus Flaccus C6, I5 I, I67

Cn. Pompeius I67

C. Sentius Saturninus C6, I07, I5 I, I67

C. Sosius I 50, I67

M. (Valerius) Messalla Messallinus C8, I 50, I 52, I 68

M. ? Valerius Potitus Messalla i 54, I66
L Vicinius? $\mathrm{AB} 9$

-]rinus? $\mathrm{AB}_{9}$

\section{Dei deaeque}

Apollo C9, 32, I39, I4I, I43

Diana C9, I0, 32, I02, I39, I 46

Hercules Victor C Io

Ilithyiae I I5, II7

Iuno 102

Iuno Regina I I 9, I 2 I, I 25

Iuppiter Optimus Maximus C ıо (Stator), 9, 30f., 3 I (Tonans), 70, I03, I05

Latona $\mathrm{C}_{9}$

Moerae 90, 92

Ops 75

Terra Mater I 34, I 36

Jam $\mathrm{C}_{9}$

]m $\mathrm{C}_{10}$

\section{Alii}

M. Iunius Silanus AB 8

C. Silanus AB 6, 52, 59

C. Furnius AB 6

L. Cestius $5 \mathrm{I}$

Cn. Cornelius Lentulus AB 8

L. Petronius Rufus $5 \mathrm{I}$

Q. Horatius Flaccus I49

\section{Loci}

Capitolium 9, I8, 30, 70, 75, 79, 81, I03, I I9, I 38, I 48

Curia Iulia AB 8

Palatium 3 I, I 39

Saepta Iulia 50

Theatrum Pompei I57, I6I

Theatrum in Circo Flaminio is 7 f., I6 I

Tiberis 90, I08, II 5, I34, I 57 
Index nominum et locorum Act. Sev.

\section{Septimius Severus}

L. Septimius Severus 2, 17, 27, 34, 4I, 55, $56,59,61,65,72,73,85,103,105$, I I 2 , I I3, 2IO, 2II, 275, 277: Severus 79, 83, 92, I45, I 55, I79, I79, I 83, I 84, 222, 223 , 229f., 229, 23I, 249, 250, 252, 254, 255, 256, 257, 266, 282; Pius 85; Imperator I 52, 282 ?, 283 ?, 287 ?; Imperator Caesar 2, 27, 34, 4I, 55, 56, 59, 6I, 65, 7I, 73, IO3, IO5, I I 2, I I 3, 209, 2 I I, 273, 277; Pius Pertinax 2, 27, 34, 4I, 55, 56, 59, 65, 72, 73, I03, IO5, I I 2, I I 3, 2 I0, 2 I I, 275, 277; Augustus 2, 27, 34, 4I, 56, 56, 65, $72,73,85,103,105$, I I3, I I3, I25?, I56, I79, I 84, 2 IO, 2 I I, 223, 229, 23 I, 255, 275, 277; Arabicus 2, 27, 34, 4I, 56, 57, $59,65,72,73$, Iо3, Iо5, I I3, I I3, 2 Iо, 2I I, 275, 277; Adiabenicus 2, 27, 34, 4I, $56,57,59,65,72,73,103,105$, I I3, II 4 , 2 I0, 2 I I, 275, 277; Parthicus maximus 2, $27,34,42,56,57,59,65,72,73,103,105$, I I 3, I I 4, 2 I0, 2 I I, 275, 277; Fortissimus 27, 34, 42; Felicissimus 27, 34, 42; Pater Patriae 27, 34, 42, 56, 60, 73, I I3, 2 I0, 276; pontifex maximus 56, 60, 72, I I 3 , I 26 ?, 2 10, 276; Imperator XI I7, I9, 56, 60, 72, I I3, 210, 276; tribunicia potestate XII 3, 56, 60, 72, I I 3, 2 I0, 276; consul III $56,60,72$, I I 3,2 I0, 276

\section{Septimius Severus et Caracalla}

Imperatores 28, 59, 79, 82, I79, 222, 228, 250, 254, 266, 282; Augusti 60, 62, 68, 68, $79,83,87, \mathrm{I79}, \mathrm{I} 8_{3}, \mathrm{I} 8_{3}, 208,223,229$, 230, 233, 249, $250,25 \mathrm{I}, 252,254,256$, 266, 268, 269, 282

\section{Caracalla}

M. Aurellius Antoninus Pius 4, I7, 2 I, 27, 42, 56, 60, 62, 65, 73, 103, 105, I I3, 2 10f., 276; Antoninus 79, 83, I65, I79, I80, I 83, 222 f., 224, 229, 230, 249, 250,252 , 254, 254, 256, 266, 282; Augustus 4, I7, 28, 42, 56, 103, I65, I 80, 2 I I, 224, 230, 254, 276; Imperator 224, 230, 254; Imperator Caesar 4, 27, 42, 56, 60, 62, 65, 73, I05, II3, 2 I0, 276; tribunicia potestate VII 73, 2 I I, 277; consul 73, 2 I I, 277

\section{Geta}

P. Septimius Geta nob. Caes. 4; Geta Caesar I80, I 8 I, I 83, 223, 224, 225, 229, 230, 249, 250, 252, 268; Geta nobilissimus Caesar 4, I7, 27, 42, 56, 73, I04, I05, II 3 , I 79, 2 I I, 266, 277, 282, 283

\section{Plautianus}

C. Fulvius Plautianus 5; praefectus praetorio $5,17,179,180,183,223,224,229$, $230,250,253,266,282$

\section{Iulia Domna}

Iulia Augusta 95, I 5 I, I78, I84, I8 5, I87, 228, 259; Mater Castrorum 95, I78, I 84, 228, 259; coniux Imperatoris I 52, I78, I 84

\section{Quindecimviri}

XVviri s. f. 5, 26, 29, 60, 62, 74, 82, 88, 100, I I4, I 3 I, I 36, I 39, I42, I62, I63, I66, I79, I80, I84, I87, 206, 2I 2, 223, 224, $224,228,229,230,253,266,268,279$, 282

Manilius Fuscus 6, 66, 208, 271, 295?

M. Spurius Verginius I4

Calpurnius Maximus 25

Antius Crescens Calpurnianus $49 \mathrm{f}$.

Atulenus Rufinus 5I, 86

Iulius Pompeius Rusonianus mag. 61, 63, 66f., 87f., IOI, I43, 207, 270

Nonius Arrius Mucianus 65f., 69, 86, 207, 272, 273

Pollienus Auspex 66, 68, 8I

Cocceius Vibianus 66, $70 \mathrm{f} ., 88$

Atulenus Rufinus 66, 69

Aiacius Modestus 66, 69, 86, I 83, 207, 272

Fabius Magnus 66, I 83

Antius Crescens Calpurnianus 67, 207f., $27 \mathrm{I}$

Cassius Pius Marcellinus 67, 89, 207, 271, 300

Venidius Rufus 67, 208, 269

Fulvius Fuscus Granianus 67f., 86f., 208, 269

Ulpius Soter 68, 8 I, 90, I 43f., 207, 255, 270

Salvius Tuscus 69, $8 \mathrm{I}$

Ofilius Macedo 70, 207, 272 
Vetina Mamertinus 87, 207, 270, $30 \mathrm{I}$

Jrnus Pr. 207, 272

Saevinius Proculus 208, 269

Gargilius Antiquus 208, 270

\section{Vates}

Sibylla 20

\section{Virgines Vestales}

Numisia Maximilla I 2 If, I 84 f.

Terentia Flavola I 22, I 85

\section{Dei deaeque}

Iuno Lucina i 8

Apollo 60, 64, 68, 80, 94, 230, 23 I, 23 I, 234

Apollo Palatinus 229

Phoebus 236

Iuppiter Optimus Maximus 93, I24, I 57 , I63, I $8325 \mathrm{I}$

Iuno Regina 93, I8 I, I8 I, I84, I85, 28 5

Iuno 228

Moerae I38, 227

Ilithyiae I 72,233

Diana 206, 228, 23I, 233

Terra Mater 225, 225

Bacchus 243

Mars? 245

\section{Divi}

Divus Marcus Antoninus Pius 55, 57, 71, 73, II2, II4, 209, 2II, 273, 278; Germanicus 55, 57, 7I, 73, II 2, II4, 209, 2I I, 273, 278; Sarmaticus 55, 57, 7I, 73, I I 2, I I 4, 209, 2 I I , 273, 278;

Divus Commodus 55, 7 I f., I I 2, 209, 274

Divus Antoninus Pius 55, 57, 72, 74, I I 2, I I 4, 209f., 2 I I , 274, 278

Divus Hadrianus 55, 57, 72, 74, I I 2, I I 4, 2 I0, 2 I 2, 274, 278

Divus Traianus 55, 57, 72, 74, I I 2, I I 4, 2 I0, 2I 2, 274, 278; Parthicus 55, 57, 72, 74, I I 2, 2 I0, 2 I 2, 274, 279

Divus Nerva 55, 57, 72, 74, I I 2, I I 4, 2 I0, 2 I 2, 275, 279

\section{Pantomimi}

Pylades 220, 222

Apolaustus 220, 22 I, 222

Marcus $22 \mathrm{I}(2), 222$

\section{Matronae}

Fl. Politta I 88

Rufria [ I 88

Statilia M[axima?] I 89

]nia Laeta I 89

$\mathrm{Ve}[\mathrm{I} 89$

]ia Pia Tiberiana 190

L[ $\cdots]$ elia[ I 90

Magia [ I90

Fufid[ia I9I

Crepere[ia I9I

P]ontia Paulina I 92

Cl. Eudaem[ I93

]la $\mathrm{I} 94$

Postumia I95

]a 196

Hort]ensia Polla Do[ 197

$\mathrm{P}] \mathrm{aca}$ [t 198

]illa 199

Vibenn[ia 199

Iulia Taria Strat[o]nice 200

Ro]mana Do[ 200

]nia Luperciana 20I

Domitia Flaccill[a $20 \mathrm{I}$

Pa]ulina 20I

$\mathrm{Ta}$ [ri]a Cornelia Asiana 202

C[l. D]ryantilla Platonis 202

Iulia Suem[ias Bassiana 202

a] Urbiana 203

Caesennia Tusidiana 203

Claudia V[al]entina 203

]elia Falviana 203

Octavia Athenais 204

Semproni[a Sp]oletina 204

Maria Passen[ia(na)] 204

Aelia Gemellina 205

Antonia Tironi[1]la 205

[A] elia Marciana 205

\section{Mariti}

Manilius (Fuscus) I 88

]atilius Severus I 88

[Cal]purnius [Maxi]mus I $88 \mathrm{f}$.

Ennius Marcianus I 89

Caecilius Aristo I 89

A?]vitus I90

P]ontius Paulinus I90

[Valerius? Ma]uricus I9I

D]emetrius I9I

]nis 193 
Attius Rufin[us I94

Festus I95

Calpurnius Front[inus I96

Valerius Crescens I99

Iul[ 200

Laberius [ 200

Ulpius Pompeian[us 20I

Ulpius Antonin[us 20I

Caesennius Servili[anus 20I

]nus 202

Nummius Faustinianus 202

Cornelius Optat[us 202

[Varius Marcellus] 202

Flavius Drusianus 203

Livius Rogatus 203

Aquilius Agri[cola] 203

Tarronius 203

Campanus trib. 204

Flavius Clemens trib. 204

Cornelius Fel[ix trib. 204

]ratus trib. 204

]inus primipil. 205

Armenius Iulianus 205

Iulius Max[imus] 205

Cossin[ius 205

]stris 205

\section{Pueri carminis saecularis}

]s Maximus 26I

$\mathrm{I}[\mathrm{ul}] \mathrm{iu}[\mathrm{s}$ F]austinus 26I

Iulius Crescens 26I

Iul. $\mathrm{Ca}[\mathrm{s}]$ sius Paulinus 26I

$\mathrm{Clo}[26 \mathrm{I}$

]m[ 26I

Alfius Avitus 26I

Opratius Ti[t]ianus 26I

Flavius Iulius Latron[ianus] 26I

]ianus 262

Vmbilius [Ma]ximinus 262

Claudius Pacatianus 262

$\mathrm{Iu}[\mathrm{l}]$ ius $\mathrm{Sa}$ [t]yrus Dryan[tianus] 262

]s Maxim[ 262

]s Vlpius Attianus Iun. 262

Laberius Pompeianus 262

Cattius Clement[inus 262

[Baburius Herc(?)]ulanius 263

Baebius Marcellinus 263

Aelius Antipater 263

Corfin[iu]s Felix $\{s\} 263$

Corn $\left[\cdot\right.$ I $\left._{5} \cdot\right]$ s eq. R 263
Licin[iu]s Aemil[ia]nus Ingen. 263

Puellae carminis saecularis

Manilia Lucana Po[llitta] 263

]lia Crispina Arrian[a] 264

[A]lfia Vestin[a] Maxi[ma] 264

[Sa]lvia Postu[mia] Varia 264

Non[ia $\cdots$ V]aleriana 264

Aemilia Iunia C[lemen]tina 264

Flavia Rom[a]na 264

Antonia [ 264

] Maxima Iuniana 265

Flavia [A]ntonia Polynic[e] 265

]lia Casta Statianilla 265

Arri[a C?]or[nelia?] Clementina 265

Corn[eli]a Claudia Pia 265

Va[leria? ...]nia Pacata 265

[B]aburia $\mathrm{Va}[265$

]a Nepotiana 266

Domitia Diotima 266

Precilia Au[g]urina 266

Pueri lusus Troiae

A]emi[1 307

S]atyrus Do[ 307

]s Regillu[s] 308

Marcius Vic[tor 308

Virius [ 309

] Musiarcus 309

Iunius [ 309

Rosc[ius 3 Io

C]ornelianus 3 Io

Clodius Mar[ 3 Io

]rus Numer[ 3 I I

]nus Aper 3 II

Ocratius Titianus 3I I

Jus 3 I 2

Bassaeus $\left[\cdot \mathrm{IO}^{\circ} \cdot\right]$ anus $3 \mathrm{I} 2$

Alfius Maximus 3 I 2

Suf [ 3 I 2

]s Silianus 3 I 3

]atianus $3 \mathrm{I} 3$

Flav[ius $\cdot 7 \cdot] \operatorname{co}[3 \mathrm{I} 3$

] Placidu[s 3 I 4

Ma]rcellinus 3 I 4

A[ 3 I 4

]omini[ 3 Is

[Ne]ratius Priscu[s] 3 I 5

$\mathrm{Bab}\left[\cdot \mathrm{I}_{2} \cdot\right]$ us $3 \mathrm{I} 6$

Vettiu[s 3 I 6 
Licini[us $\cdot$ Io e e]q. R. fil. 317

$\mathrm{C}[317$

\section{Loci}

Curia Iulia 5

Palatium 49, 60, 64, 68, 79, I79, 223, 229, $25 \mathrm{I}, 282$

Roma Quadrata 69

Tarentum IOI

Terentum 223

Via Sacra I 8 f, 249
Odium I 20, 270

Capitolium I78, I79, 250

Tiberis 67, I20, 208, 223, 269

Theatrum Pompeianum 2 I 3,267

Theatrum Pompei 220, 22I, 222, $27 \mathrm{I}$

Odium 2I3, 22 I, 222, 267

Circus Maximus 2 I 4

Roma 239

Forum Romanum 249

Arcus Severi 249

Porticus [ II9 